\title{
Instabilities of vortices in binary mixture of trapped Bose-Einstein condensates: Role of collective excitations with positive and negative energies
}

\author{
Dmitry V. Skryabin * \\ Department of Physics and Applied Physics, University of Strathclyde, Glasgow, G4 ONG, United Kingdom
}

(19 January, 2000; to appear in Phys. Rev. A, January 2001)

\begin{abstract}
Correspondence between frequency and energy spectra and biorthogonality conditions for the excitations of Bose-Einstein condensates described by Gross-Pitaevskii model have been derived selfconsistently revealing several novel aspects originating in nonselfadjoitness of the Bogoliubov operator. It has been demonstrated that frequency resonances of the excitations with positive and negative energies can lead to their mutual annihilation and appearance of the collective modes with complex frequencies and zero energies. Conditions for the avoided crossing of energy levels have also been discussed. General theory has been verified both numerically and analytically in the weak interaction limit considering an example of vortices in a binary mixture of condensates. Growth of excitations with complex frequencies leads to spiraling of the unit and double vortices out of the condensate center to its periphery and to splitting of the double and higher order vortices to the unit ones.
\end{abstract}

\section{INTRODUCTION}

Recent observations of the quantized vortices in a dilute ultracold gas of ${ }^{87} R b$ atoms [1] 2] are spectacular evidences of the superfluid properties of atomic gases below transition temperature for formation of Bose-Einstein condensate (BEC). First experimental results were obtained, using method suggested by Williams and Holland [3], in a binary mixture of the hyperfine states of ${ }^{87} R b$ [1] and more recent experiments [2] have demonstrated vortices and vortex lattices in an optically stirred single component condensate.

Gross-Pitaevskii (GP) equation [A] is a widely used approximation to describe dynamics of the quantized vortices in the superfluid component of Bose gases at temperatures below critical. The GP equation falls into a general class of Hamiltonian nonlinear systems. The theory of stability of stationary solutions (equilibria) of such systems is well established at the moment, see e.g. [5]. However, a certain gap between formal mathematical knowledge and applications to physical examples still exists and BEC field is not an exception. One of the controversial examples in the BEC context is complex or imaginary eigenfrequencies in the spectrum of elementary excitations. Existence of these frequencies was first pointed out by Bogoliubov in his original work 6, for more recent references see, e.g. [7 12]. Unjustified negligence by the nonselfadjoitness of the Bogoliubov equations often lead to the association between frequencies and energies in a way standard for quantum mechanics based on selfadjoint operators, thus admitting possibility of complex energies in a conservative system.

However, rigorously proved theorem allowing clear physical interpretation of complex frequencies is known from the general theory of Hamiltonian systems [5]. It states that excitations with complex frequencies can appear only as a result of the resonance between two excitations with positive and negative energies [13. The roots of this theorem go back to 19th century [14] and it has been used for interpretation of instabilities with complex eigenvalues in plasma physics [15] and fluid dynamics [16]. Motivated by recent experiments on observation of vortices in binary mixture of condensates [1] we will demonstrate that these vortices can have complex frequencies in their spectrum, thereby giving good practical ground for selfconsistent theoretical interpretation of this phenomenon in the BEC context.

Properties of vortices in the single component magnetically trapped ultracold gases have been subject to the intensive theoretical investigations, see e.g. [9,17-32], which significantly extended classical works [4, 33] dealing with spatially unbounded case. The properties of unit vortices in the two component condensates have also been studied, in parallel and independently from this work, by Garcia-Ripoll and Pérez-Garcia 10,11. Richness of the dynamics of the two-condensate system and different approaches to the problem have led only to few overlaps which are outlined where appropriate.

In the next section we introduce coupled GP equations and briefly describe their general properties. Then, in section III, we derive Bogoliubov equations for excitations, clearly specifying differences between frequency and energy spectra of the excitations. We also explain scenario of appearance of the excitations with complex frequencies and show that they have zero energies. In section IV we verify validity of general results presented in Sec. III using perturbation

${ }^{*}$ URL: www://http.cnqo.strath.ac.uk/ dmitry 
theory in the limit of weak interaction and direct numerical study of Bogoliubov equations. In Sections IV, V we describe how long term dynamics of unit and higher order vortices can be interpreted using linear Bogoliubov theory.

\section{GROSS-PITAEVSKII EQUATIONS}

Studies of superfluid mixtures using coupled GP equations have long history and attracted significant recent activities, see e.g. [1], [7, 8, 10, 11, 34 38] and references therein. Following these works we assume that wave functions $\psi_{1,2}$ of two-species condensate inside an axial harmonic trap obey equations

$$
\begin{aligned}
& i \hbar \partial_{t} \psi_{1}=-\frac{\hbar^{2}}{2 m} \vec{\nabla}^{2} \psi_{1}+\frac{1}{2} m \Omega^{2}\left(r^{2}+\sigma^{2} z^{2}\right) \psi_{1} \\
& +\left(u_{11}\left|\psi_{1}\right|^{2}+u_{12}\left|\psi_{2}\right|^{2}\right) \psi_{1}, \\
& i \hbar \partial_{t} \psi_{2}=-\frac{\hbar^{2}}{2 m} \vec{\nabla}^{2} \psi_{2}+\frac{1}{2} m \Omega^{2}\left(r^{2}+\sigma^{2} z^{2}\right) \psi_{2} \\
& +\left(u_{22}\left|\psi_{2}\right|^{2}+u_{21}\left|\psi_{1}\right|^{2}\right) \psi_{2},
\end{aligned}
$$

where for simplicity we have neglected by possible differences of atomic masses $m_{1,2}=m$ and trap frequencies, $\Omega_{1,2}=\Omega, \sigma_{1,2}=\sigma, \vec{\nabla}=\vec{i}_{x} \partial_{x}+\vec{i}_{y} \partial_{y}+\vec{i}_{z} \partial_{z}, r^{2}=x^{2}+y^{2}$. Coefficients $u_{i j}=4 \pi \hbar^{2} a_{i j} / m$ characterise intra- and inter-species interaction with corresponding two-body scattering lengths $a_{11} \neq a_{22}$ and $a_{12}=a_{21}$.

At this point we introduce dimensionless time and space variables $\tilde{t}=\Omega t$ and $(\tilde{x}, \tilde{y}, \tilde{z})=(x, y, z) / a_{h o}$ and normalisation for the wave functions $\tilde{\psi}_{1,2}=a_{h o}^{3 / 2} \psi_{1,2} / \sqrt{N_{1}}$, where $a_{h o}=\sqrt{\hbar /(m \Omega)}$ is the harmonic oscillator strength and $N_{1,2}=\int d V\left|\psi_{1,2}\right|^{2}$ are the numbers of particles. We will consider the quasi-2D model to simplify our numerical study. This approximation was previously used in several works, see e.g. [9 11], and it is applicable not only for pancake traps, $\sigma \gg 1$, but also captures main qualitative features of spherical traps. To make further reduction of Eqs. (1) we redefine the wave functions once more:

$$
\tilde{\psi}_{1,2}=\left[\frac{\sigma}{2 \pi}\right]^{1 / 4} \Psi_{1,2}(\tilde{x}, \tilde{y}, \tilde{t}) e^{-\sigma \tilde{z}^{2} / 4} e^{-i \mu_{1,2} \tilde{t}-i \sigma \tilde{t} / 2},
$$

where $\mu_{1,2}$ are the chemical potentials. Dropping tilde we find that equations for $\Psi_{1,2}$ and $\Psi_{1,2}^{*}$ can be put into Hamiltonian form

$$
\begin{gathered}
i \partial_{t} \vec{\psi}+\hat{\eta} \frac{\delta H}{\delta \vec{\psi}^{*}}=0, \\
\vec{\psi}=\left[\begin{array}{c}
\Psi_{1} \\
\Psi_{1}^{*} \\
\Psi_{2} \\
\Psi_{2}^{*}
\end{array}\right], \hat{\eta}=\left[\begin{array}{cccc}
-1 & 0 & 0 & 0 \\
0 & 1 & 0 & 0 \\
0 & 0 & -1 & 0 \\
0 & 0 & 0 & 1
\end{array}\right], \frac{\delta H}{\delta \vec{\psi}^{*}}=\left[\begin{array}{c}
\delta H / \delta \Psi_{1}^{*} \\
\delta H / \delta \Psi_{1} \\
\delta H / \delta \Psi_{2}^{*} \\
\delta H / \delta \Psi_{2}
\end{array}\right], \\
H=\int d x d y\left(\left|\vec{\nabla} \Psi_{1}\right|^{2}+\left|\vec{\nabla} \Psi_{2}\right|^{2}\right. \\
+\left(\hat{V}-\mu_{1}\right)\left|\Psi_{1}\right|^{2}+\left(\hat{V}-\mu_{2}\right)\left|\Psi_{2}\right|^{2} \\
\left.+\frac{g}{2}\left[\beta_{11}\left|\Psi_{1}\right|^{4}+\beta_{22}\left|\Psi_{2}\right|^{4}+2 \beta_{12}\left|\Psi_{1}\right|^{2}\left|\Psi_{2}\right|^{2}\right]\right) .
\end{gathered}
$$

where $H$ is the energy functional (or Hamiltonian), $\vec{\nabla}=\vec{i}_{x} \partial_{x}+\vec{i}_{y} \partial_{y}, \hat{V}=r^{2} / 4$ is the harmonic potential, $g$ is the interaction parameter $g=8 \sqrt{\pi \sigma} N_{1} a_{11} / a_{h o}, \beta_{12}=a_{12} / a_{11}$, and $\beta_{22}=a_{22} / a_{11} \cdot \beta_{11}=1$ and it is left in the equations for the sake of the symmetry.

Invariancies of $H$ with respect to the infinitesimal rotations and two parameter phase transformation

$$
\left(\Psi_{1}, \Psi_{2}\right) \rightarrow\left(\Psi_{1} e^{i \phi_{1}}, \Psi_{2} e^{i \phi_{2}}\right),
$$

result in conservation of the total angular momentum and of the total number of particles in each component.

Radially symmetric stationary states of the condensate (equilibria) can be presented in the form

$$
\Psi_{j}=A_{j}(r) e^{i L_{j} \theta}, j=1,2
$$


where $\theta$ is the polar angle and $A_{j}$ are real functions. Using method suggested in [1,3] only states with vortex in one of the components can be created and therefore we will consider below only cases with $L_{2}>0$ and $L_{1}=0$. Functions $A_{j}(r)$ were found numerically using Newton method. Chemical potentials $\mu_{1,2}$ were found from the normalization conditions

$$
2 \pi \int r d r A_{1}^{2}=1,2 \pi \int r d r A_{2}^{2}=\frac{N_{2}}{N_{1}} \equiv N
$$

\section{FREQUENCY AND ENERGY SPECTRA OF COLLECTIVE EXCITATIONS}

\section{A. Bogoliubov equations and frequency spectrum}

To study spectrum of BEC at equilibrium we linearise Eqs. (3) using substitutions

$$
\Psi_{j}=\left(A_{j}(r)+f_{j}(r, \theta, t)\right) e^{i L_{j} \theta},
$$

where $f_{j}$ are small and complex. Assuming that excitation are periodic in $\theta$ with period $2 \pi$ we expand $f_{j}$ into Fourier series:

$$
f_{j}=\sum_{l}\left(U_{l j}(r, t) e^{i l \theta}+V_{l j}^{*}(r, t) e^{-i l \theta}\right)
$$

where $l=0, \pm 1, \pm 2, \ldots$ Then

$$
i \partial_{t} \vec{W}_{l}+\hat{\eta} \hat{\mathcal{H}}_{l} \vec{W}_{l}=0
$$

is the set of linear partial differential equations resulting from the substitution of (8), (9) into Eq. (3). Here $\vec{W}_{l}=\left(U_{l 1}, V_{l 1}, U_{l 2}, V_{l 2}\right)^{T}$,

$$
\hat{\mathcal{H}}_{l}=\left[\begin{array}{cccc}
\hat{\mathcal{L}}_{l, 1} & g \beta_{11} A_{1}^{2} & g \beta_{12} A_{1} A_{2} & g \beta_{12} A_{1} A_{2} \\
g \beta_{11} A_{1}^{2} & \hat{\mathcal{L}}_{-l, 1} & g \beta_{12} A_{1} A_{2} & g \beta_{12} A_{1} A_{2} \\
g \beta_{12} A_{1} A_{2} & g \beta_{12} A_{1} A_{2} & \hat{\mathcal{L}}_{l, 2} & g \beta_{22} A_{2}^{2} \\
g \beta_{12} A_{1} A_{2} & g \beta_{12} A_{1} A_{2} & g \beta_{22} A_{2}^{2} & \hat{\mathcal{L}}_{-l, 2}
\end{array}\right],
$$

is a self-adjoint operator and

$$
\begin{aligned}
& \hat{\mathcal{L}}_{l, j}=-\frac{1}{r} \frac{\partial}{\partial r} r \frac{\partial}{\partial r}+\frac{1}{r^{2}}\left(L_{j}+l\right)^{2}+\hat{V}-\mu_{j} \\
& +2 g \beta_{j j} A_{j}^{2}+g \beta_{12} A_{j^{\prime}}^{2} ; j=1,2 ; j^{\prime}=2,1 .
\end{aligned}
$$

Frequency spectra of $\hat{\eta} \hat{\mathcal{H}}_{l}$ are discrete, providing $\hat{V} \neq 0$, therefore phonons, strictly speaking, are absent in the trap geometry. In accord with standard terminology [a [39], all spatially bounded elementary excitations can be called collective excitations (or collective modes) of an equilibrium under consideration. Linearised equations for excitations in a Bose gas, similar to Eq. (10), were first derived by Bogoliubov and expansion (9) was first applied in the context of the vortex excitations by Pitaevskii [4]. To find frequency spectrum and collective modes we need to solve set of the eigenvalue problems

$$
\hat{\eta} \hat{\mathcal{H}}_{l} \vec{w}_{l n}=\omega_{l n} \vec{w}_{l n} .
$$

$\hat{\eta} \hat{\mathcal{H}}_{l}$ are non-self-adjoint operators and therefore complex frequencies are not forbidden. If $\vec{w}_{l n}$ is an eigenvector of $\hat{\eta} \hat{\mathcal{H}}_{l}$ with eigenvalue $\omega_{l n}$ it is selfevident that $\vec{w}_{l n}^{*}$ is also an eigenvector with eigenvalue $\omega_{l n}^{*}$ and it can be shown that $\hat{\eta} \hat{\mathcal{H}}_{-l}$ has eigenvectors $\vec{w}_{-l n}=\hat{\tau} \vec{w}_{l n}$ and $\vec{w}_{-l n}^{*}$ with eigenvalues $-\omega_{l n}$ and $-\omega_{l n}^{*}$, respectively. Here

$$
\hat{\tau}=\left[\begin{array}{llll}
0 & 1 & 0 & 0 \\
1 & 0 & 0 & 0 \\
0 & 0 & 0 & 1 \\
0 & 0 & 1 & 0
\end{array}\right] .
$$


Thus spectrum of $\hat{\eta} \hat{\mathcal{H}}_{-l}$ can be obtained by reflection of the spectrum of $\hat{\eta} \hat{\mathcal{H}}_{l}$ with respect to the line Rew $=0$ in the plane $(R e \omega, \operatorname{Im} \omega)$. In other words, it means that purely real or purely imaginary frequencies of the elementary excitation exist in pairs and complex frequencies exist in quartets and that

$$
\operatorname{Tr}\left\{\hat{\eta}\left(\hat{\mathcal{H}}_{l}+\hat{\mathcal{H}}_{-l}\right)\right\}=\sum_{n}\left(\omega_{l n}+\omega_{-l n}\right)=0 .
$$

Any equilibrium state of the condensate is spectrally stable [5] if its spectrum is real. If there is at least one frequency with negative imaginary part then corresponding collective mode will grow in time destabilising the equilibrium, which is called spectrally unstable.

\section{B. Biorthogonality}

Eigenmodes of $\hat{\eta} \hat{\mathcal{H}}_{l}$ are biorthogonal to the modes of the adjoint eigenvalue problem [40], i.e.

$$
\left\langle\vec{w}_{l n}, \vec{a}_{l n^{\prime}}\right\rangle=0
$$

where $n \neq n^{\prime}$ and $\vec{a}_{l n}$ obey

$$
\left(\hat{\eta} \hat{\mathcal{H}}_{l}\right)^{\dagger} \vec{a}_{l n}=\hat{\mathcal{H}}_{l} \hat{\eta} \vec{a}_{l n}=\omega_{l n}^{*} \vec{a}_{l n},
$$

and $\langle\vec{b}, \vec{c}\rangle=2 \pi \sum_{k} \int_{0}^{\infty} r d r b_{k}^{*} c_{k}$ for any $\vec{b}, \vec{c}$. Factor $2 \pi$ is introduced to mimic integration over $\theta$. The key feature of our model, originating in its Hamiltonian structure, is that transformation linking $\vec{w}_{l n}$ and its adjoint $\vec{a}_{l n}$ can be found in explicit and simple form. If $\vec{w}_{l n}$ is an eigenmode of $\hat{\eta} \hat{\mathcal{H}}_{l}$ with frequency $\omega_{l n}$ then it can be checked that $\hat{\eta} \vec{w}_{l n}^{*}$ and $\hat{\eta} \vec{w}_{l n}$ are eigenmodes of $\hat{\mathcal{H}}_{l} \hat{\eta}$ with eigenvalues, respectively, $\omega_{l n}^{*}$ and $\omega_{l n}$. The mode adjoint to $\vec{w}_{l n}$ is $\hat{\eta} \vec{w}_{l n}^{*}$, therefore if $I m \omega_{l n} \neq 0$ then biorthogonality condition (13) implies

$$
\left\langle\vec{w}_{l n}, \hat{\eta} \vec{w}_{l n}\right\rangle=0 .
$$

If $\omega_{l n}$ is real, then $\vec{w}_{l n}$ is also real and $\left\langle\vec{w}_{l n}, \hat{\eta} \vec{w}_{l n}\right\rangle \neq 0$. Normalisation constant can always be chosen in such a way that

$$
\left|\left\langle\vec{w}_{l n}, \hat{\eta} \vec{w}_{l n}\right\rangle\right|=2 .
$$

Convenience of making the left hand side of Eq. (16) equal to 2 will become clear below, when Eqs. (19), (20) for energies of elementary excitations are derived. Eq. (16) makes it explicit that inner product $\left\langle\vec{w}_{l n}, \hat{\eta} \vec{w}_{l n}\right\rangle$ can be either positive or negative. This point often remains silent if one derives conditions similar to (16) as part of the diagonalisation procedure of the second-quantised Hamiltonian disregarding eigenmodes with negative and zero values of $\left\langle\vec{w}_{l n}, \hat{\eta} \vec{w}_{l n}\right\rangle$ [33]. The fact that inner product of a real eigenmode with its adjoint can be negative, is different from standard quantum mechanics based on the selfadjoint operators. These difference can have series of consequences and one of them is that energy levels are not necessarily linked to the eigenfrequencies according to the standard rule $\hbar \omega$, see below. Note, that origin of the nonselfadjoitness in our case is the nonlinearity of GP equations. If particles in the condensate do not interact, $g=0$, then $\hat{\eta} \hat{\mathcal{H}}_{l}$ become diagonal and self-adjoint.

\section{Energy spectrum}

As a prelude to calculation of energies of the elementary excitations it is instructive to introduce notion of the nonlinear stability, i.e. stability under the full nonlinear dynamics. According to Dirichlet's theorem [5], nonlinear stability is ensured if an equilibrium state under consideration is either minimum or maximum of the functional $H$. Note here, that excitations change number of particles in the equilibrium state, therefore it was convenient to introduce energy functional $H$, which is actually the so-called modified energy [33, i.e. it is the energy functional for Eq. (1) modified by addition of the number of particles integrals, see terms proportional to $\mu_{j}$ in Eq. (5). Let us assume that $\vec{w}_{l}(r) e^{i l \theta}$ is a small initial perturbation of an equilibrium state of the condensate, then

$$
H=H_{0}+\frac{1}{2}\left\langle\vec{w}_{l}, \hat{\mathcal{H}}_{l} \vec{w}_{l}\right\rangle+\ldots,
$$

where $H$ is the energy of the perturbed equilibrium and $H_{0}$ is calculated at the exact equilibrium. The equilibrium is nonlinearly stable if eigenvalue problems 


$$
\hat{\mathcal{H}}_{l} \vec{\beta}_{l m}=\alpha_{l m} \vec{\beta}_{l m}
$$

have all their eigenvalues either negative or positive, except zero eigenvalues generated by continuous symmetries. Spectral instability implies nonlinear one, and nonlinear stability implies spectral one, but not vise versa [5]. In the nonrotating traps all the higher order states of the condensate including vortices are not the local extrema of the energy [19,23,25,27]. Let us stress, however, that their nonlinear instability can not be guaranteed by this fact alone and requires separate consideration.
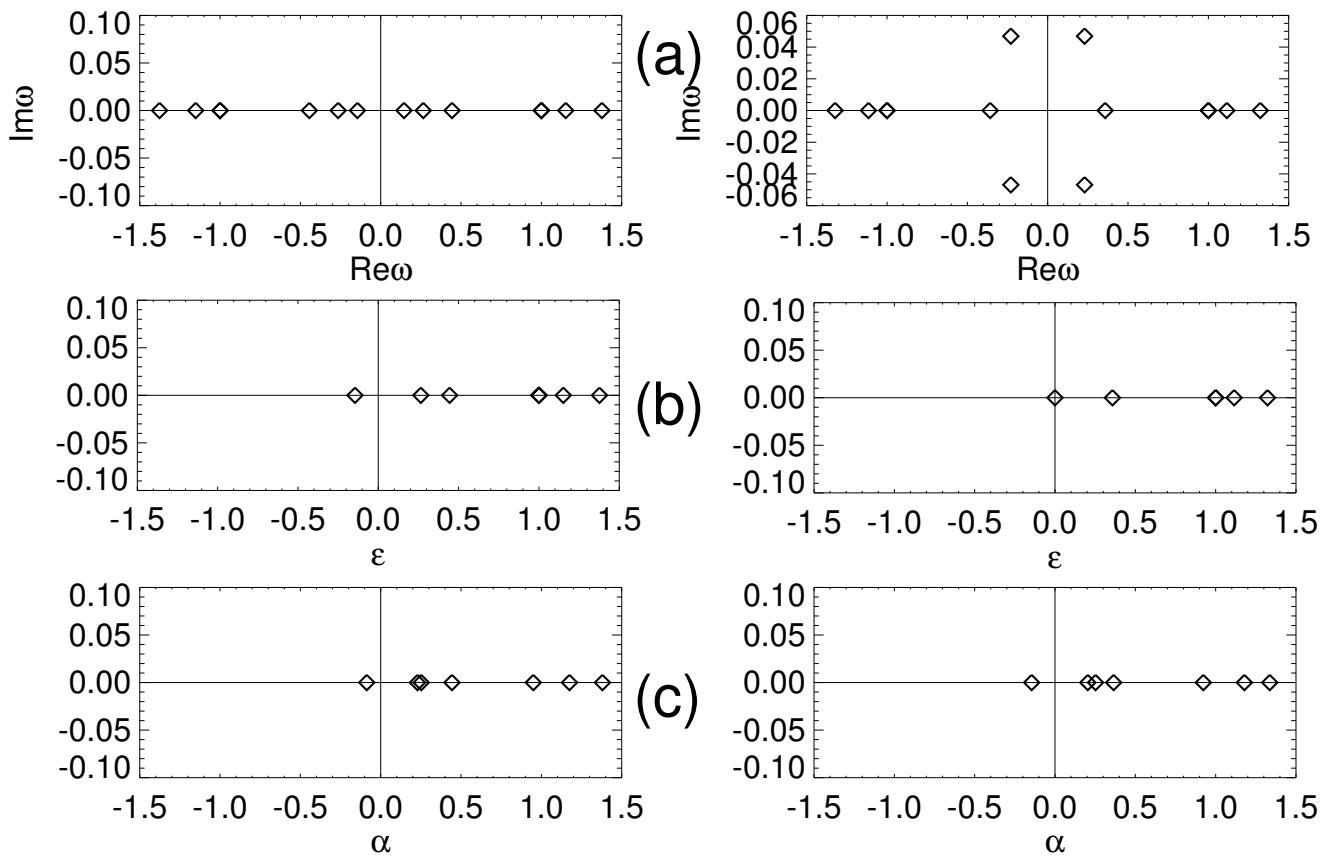

FIG. 1. Numerically calculated frequency (a) and energy (b) spectra corresponding to the collective modes with $l= \pm 1$ of the spectrally stable (left panel) and spectraly unstable (right panel) unit vortices: $L_{1}=0, L_{2}=1, g=250, N=1$, other parameters for the left/right panel correspond to the vortex in the state 1/state 2, see text after Eq. (32). The correspondence between frequency and energy spectra is obvious, see Eqs. 20,21). (c) Spectrum of the eigenvalue problems $\hat{\mathcal{H}}_{ \pm 1} \vec{\beta}_{n}=\alpha_{n} \vec{\beta}_{n}$; parameters as for (a),(b).

If $\vec{w}_{l}$ in Eq. (17) is an eigenmode $\vec{w}_{l n}$ of $\hat{\eta} \hat{\mathcal{H}}_{l}$ then

$$
\epsilon_{l n}=\frac{1}{2}\left\langle\vec{w}_{l n}, \hat{\mathcal{H}}_{l} \vec{w}_{l n}\right\rangle=\frac{1}{2} \omega_{l n}\left\langle\vec{w}_{l n}, \hat{\eta} \vec{w}_{l n}\right\rangle
$$

measures energy of this mode. Assuming that $\omega_{l n}$ is real and using biorthogonality conditions (16) one gets

$$
\epsilon_{l n}=\omega_{l n} \operatorname{sign}\left\langle\vec{w}_{l n}, \hat{\eta} \vec{w}_{l n}\right\rangle
$$

If $I m \omega_{l n} \neq 0$ then Eq. (15) implies

$$
\epsilon_{l n}=0 .
$$

Energy in physical units is given by $\epsilon_{l n} \hbar \Omega$.

It is readily demonstrated that

$$
\left\langle\vec{w}_{l n}, \hat{\eta} \vec{w}_{l n}\right\rangle=-\left\langle\hat{\tau} \vec{w}_{l n}, \hat{\eta} \hat{\tau} \vec{w}_{l n}\right\rangle=-\left\langle\vec{w}_{-l n}, \hat{\eta} \vec{w}_{-l n}\right\rangle,
$$

therefore modes with frequencies $\omega_{l n}$ and $\omega_{-l n}=-\omega_{l n}$ have their energies equal in sign and value.

Eigenfunctions $\vec{\beta}_{m l}$ are orthogonal $\left\langle\vec{\beta}_{l m}, \vec{\beta}_{l m^{\prime}}\right\rangle=\delta_{m m^{\prime}}$ and form complete basis. Therefore $\vec{w}_{l n}$ in Eq. (19) can be expanded in terms of $\vec{\beta}_{l m}$, which gives $\epsilon_{l n}=\sum_{m} \alpha_{l m}\left\langle\vec{w}_{l n}, \vec{\beta}_{l m}\right\rangle^{2}$. Thus an equilibrium can have collective modes carrying energy with opposite signs only if it is not a local extremum of the energy functional. 


\section{Resonances and instabilities, crossings and avoided crossings}

Instabilities with imaginary eigenvalues have been found to dominate dynamics of homogeneous BEC with attractive interaction [6] and mixture of two condensates with repulsive interaction [7,8]. Higher order vortices and dark solitons in single component trapped condensates can be unstable with respect to modes with complex frequencies [9, 12]. In our problem instabilities with complex eigenvalues are the most important ones also. Therefore it is desirable to have criterion or theorem allowing interpretation and prediction of these instabilities. Such theorem is actually known from the general theory of the Hamiltonian dynamical systems [5] and, in our context, it can be reformulated as: (i) Sign of the energy of a collective excitation preserves as parameters vary as long as there is no frequency resonance with another excitation. (ii) Condensate at equilibrium can lose spectral stability as parameters vary only in two ways: either via frequency resonance of two elementary excitations with positive and negative energies or by resonance at zero frequency. Proof of these results [5] is based on the fact, that transition from spectral stability to instability can not violate energy conservation law: $\partial_{t} H=0$.

From this theorem and preceding considerations one can conclude that complex eigenvalues in the spectrum of the vortices in Bose-Einstein condensate can appear only due to mutual annihilation of the collective excitations with positive and negative energies. The latter can coexist only for nonlinearly unstable equilibria. The theorem does not forbid for frequencies of two excitations with either the same or opposite signs of the energies simply cross each other without change of the spectral stability. In our example it typically happens when corresponding eigenmodes remain orthogonal at the exact resonance. If, however, eigenmodes of the colliding excitations start to compete for the same direction in the functional phase space and become degenerate at the resonance then crossing is not a generic scenario. If the signs of the energies of the excitations are opposite, then quartet of complex frequencies appears upon passing the resonance. Alternatively, if the signs are the same, the exact resonance can not be achieved and it becomes replaced by the so-called avoided crossing of the energy levels [5].

Figs. 1(a),(b) show numerically calculated frequency and energy spectra of the collective excitations with $l= \pm 1$ for spectrally stable (left panel) and spectrally unstable (right panel) unit vortices $\left(L_{1}=0, L_{1}=1\right)$. The negative energy excitation is clearly seen in the spectrally stable situation. At the transition threshold to spectral instability this excitation and another one, having energy with the same absolute value but opposite sign, annihilate each other. This transition is accompanied by appearance of the zero energy excitations. Examples of frequency and energy evolution under the parameter variation resulting in instabilities, crossings and avoided crossing are given in the section IV. Figs. 1(c) show spectra of $\hat{\mathcal{H}}_{ \pm 1}$ illustrating that there are no qualitative changes in these spectra after appearance of complex frequencies.

Possibility of observation of the collective modes with complex frequencies has caused some concerns and discussions [12,27. However, initial perturbations of the equilibrium state having nonzero projections on the adjoint mode $\hat{\eta} \vec{w}_{l n}^{*}$ with $I m \omega_{l n}^{*}>0$ will lead to the ultimate growth of the corresponding mode $\vec{w}_{l n}$ because it has $\operatorname{Im} \omega_{l n}<0$. The consequences of this growth is in any way diminished by the fact that the energy of this mode is zero. Wealth of references and examples of instabilities with complex eigenvalues existing in other physical contexts can be found in [5.,43].

\section{E. Goldstone and dipole modes}

Infinitesimal variations of $\phi_{j}$, see Eq. (5), generate two zero-energy eigenmodes (Goldstone modes) $\left(A_{1},-A_{1}, 0,0\right)^{T}$ and $\left(0,0, A_{2},-A_{2}\right)^{T}$ belonging to the null-eigenspace of $\hat{\eta} \hat{\mathcal{H}}_{0}$. Harmonic trapping modifies spectrum in such a way that $\hat{\eta} \hat{\mathcal{H}}_{ \pm 1}$ acquire couple of parameter independent eigenvalues $\omega= \pm 1$ with eigenfunctions

$$
\vec{w}_{ \pm 1 d}=\left[\begin{array}{c}
\omega\left(\frac{d A_{1}}{d r} \mp \frac{1}{r} L_{1} A_{1}\right)+\frac{1}{2} r A_{1} \\
\omega\left(\frac{d A_{1}}{d r} \pm \frac{1}{r} L_{1} A_{1}\right)-\frac{1}{2} r A_{1} \\
\omega\left(\frac{d A_{2}}{d r} \mp \frac{1}{r} L_{2} A_{2}\right)+\frac{1}{2} r A_{2} \\
\omega\left(\frac{d A_{2}}{d r} \pm \frac{1}{r} L_{2} A_{2}\right)-\frac{1}{2} r A_{2}
\end{array}\right]
$$

where $\omega$ can take values \pm 1 for both eigenmodes. $\vec{w}_{ \pm 1 d}$ are often called dipole modes and $\epsilon_{ \pm 1 d}=1$. Eqs. (23) generalise expressions previously derived for single-species condensates [23]. Existence of the dipole modes can also be associated with the Kohn's theorem [41,42]. 


\section{COLLECTIVE EXCITATIONS OF UNIT VORTICES: $L_{1}=0, L_{1}=1$}

We begin our analysis considering weakly interacting condensate: $g \ll 1, N \sim 1$. In this limit potential energy due to harmonic trapping $\hat{V}$ strongly dominates over the interaction energy, which allows to make explicit calculations of the frequency and energy spectra of the elementary excitations. Calculations of the excitation spectra of the vortices and dark solitons in the singly component weakly interacting condensates have been recently done by several groups of authors [12,25]. However, these calculations lack analysis of the energy sign in a sense explained in the preceding section.

We substitute asymptotic expansions $\mu_{1,2}=\mu_{1,2}^{(0)}+g \mu_{1,2}^{(1)}+O\left(g^{2}\right), A_{1,2}=A_{1,2}^{(0)}+g A_{1,2}^{(1)}+O\left(g^{2}\right)$ into the stationary $\left(\partial_{t}=0\right)$ version of Eqs. (3) and derive recurrent system of linear equations. In the zero approximation we have two uncoupled harmonic oscillator problems with eigenmodes

$$
A_{1}^{(0)}=\frac{1}{\sqrt{2 \pi}} e^{-r^{2} / 4}, A_{2}^{(0)}=\sqrt{\frac{N}{\pi}} \frac{r}{2} e^{-r^{2} / 4} .
$$

Using solvability condition of the first order problem we find asymptotic expressions for the chemical potentials: $\mu_{1}=1+g\left(2 \beta_{11}+n \beta_{12}\right) /(8 \pi)+O\left(g^{2}\right), \mu_{2}=2+g\left(n \beta_{22}+\beta_{12}\right) /(8 \pi)+O\left(g^{2}\right)$.

Then we expand operators $\hat{\mathcal{H}}_{l}$, eigenmodes $\vec{w}_{l}$ and frequencies $\omega_{l}$ into the series $\hat{\mathcal{H}}_{l}=\hat{\mathcal{H}}_{l}^{(0)}+g \hat{\mathcal{H}}_{l}^{(1)}+O\left(g^{2}\right)$, $\vec{w}_{l}=\vec{w}_{l}^{(0)}+g \vec{w}_{l}^{(1)}+O\left(g^{2}\right), \omega_{l}=\omega_{l}^{(0)}+g \omega_{l}^{(1)}+O\left(g^{2}\right)$. After substitution into Eq. (11) in the first order we find standard equation

$$
\left(\hat{\eta} \hat{\mathcal{H}}_{l}^{(0)}-\omega_{l}^{(0)}\right) \vec{w}_{l}^{(1)}=\left(\omega_{l}^{(1)}-\hat{\eta} \hat{\mathcal{H}}_{l}^{(1)}\right) \vec{w}_{l}^{(0)}
$$

$\hat{\eta} \hat{\mathcal{H}}_{l}^{(0)}$ is diagonal and self-adjoint and all its eigenmodes and eigenvalues can be found explicitly. Then using solvability condition for Eq. (25) one can find corrections for all frequencies and coefficients in the linear superposition of the zero approximation eigenmodes. Computer algebra makes technical realization of this plan by a straightforward exercise. We will present and analyse explicit analytical results only for the operator $\hat{\eta} \hat{\mathcal{H}}_{-1}$ considering vicinity of the spectral point $\omega=1$, because it contains information about origin of spectral instabilities of unit vortices. Equivalent analysis of $\hat{\eta} \hat{\mathcal{H}}_{1}$ near $\omega=-1$ gives the same results.

$\hat{\eta} \hat{\mathcal{H}}_{-1}^{(0)}$ has three unit frequencies, $\omega_{-1}^{(0)}=1$. Corresponding eigenmodes are

$$
\begin{aligned}
& \vec{b}_{1}=(0,1,0,0)^{T} \frac{r}{2 \sqrt{\pi}} e^{-r^{2} / 4} \\
& \vec{b}_{2}=(0,0,1,0)^{T} \frac{1}{\sqrt{2 \pi}} e^{-r^{2} / 4}, \\
& \vec{b}_{3}=(0,0,0,1)^{T} \frac{r^{2}}{4 \sqrt{\pi}} e^{-r^{2} / 4} .
\end{aligned}
$$

Solvability conditions for Eq. (25) lead to the characteristic determinant of the three by three matrix. We find that one of the three frequencies is associated with dipole mode $\vec{w}_{-1 d}$ and that the other two are

$$
\begin{aligned}
& \omega_{-1}^{ \pm}=1+\frac{g}{32 \pi}\left(-3 \beta_{12}-N \beta_{22} \pm \sqrt{R}\right)+O\left(g^{2}\right) \\
& R \equiv\left(3 \beta_{12}+N \beta_{22}\right)^{2}-8 \beta_{12}^{2}(N+1) .
\end{aligned}
$$

Corresponding unnormalised eigenmodes are

$$
\begin{aligned}
\vec{w}_{-1}^{ \pm}= & \sqrt{2 N}\left(N \beta_{22}-\beta_{12} \pm \sqrt{R}\right) \vec{b}_{1} \\
& +\sqrt{2}\left(3 N \beta_{22}+[1-4 N] \beta_{12} \mp \sqrt{R}\right) \vec{b}_{2} \\
& +4 N\left(\beta_{12}-\beta_{22}\right) \vec{b}_{3}+O(g) .
\end{aligned}
$$

Pair of frequencies (27) becomes complex if $R<0$, signaling spectral instability of the unit vortex. If $R>0$, then

$$
\begin{aligned}
& \left\langle\vec{w}_{-1}^{ \pm}, \hat{\eta} \vec{w}_{-1}^{ \pm}\right\rangle= \\
& 4\left[R(N-1) \pm \sqrt{R}\left\{(1-5 N) \beta_{12}+N(3+N) \beta_{22}\right\}\right]
\end{aligned}
$$


and one can check that

$$
\epsilon_{-1}^{ \pm}= \pm \omega_{-1}^{ \pm}
$$

i.e. modes with frequencies $\omega_{-1}^{+}$and $\omega_{-1}^{-}$have, respectively, positive and negative energies, see Fig. 2(c). One can also verify biorthogonality $\left\langle\vec{w}_{-1}^{ \pm}, \hat{\eta} \vec{w}_{-1}^{ \pm}\right\rangle=0=\epsilon_{-1}^{ \pm}$for $R<0$, see Eq. (15), and $\left\langle\vec{w}_{-1}^{ \pm}, \hat{\eta} \vec{w}_{-1}^{\mp}\right\rangle=0$ for $R>0$, see Eq. (16).

Assuming $\beta_{i j}>0$ and rewriting instability condition $R<0$ in the form

$$
\frac{\beta_{22}}{\beta_{12}}<\frac{1}{N}(2 \sqrt{2(N+1)}-3)
$$

one can see that unit vortices are more stable if intraspecies interaction of atoms in the vortex containing part of the condensate is somewhat larger than interspecies interaction. The choices of scattering lengths corresponding to the experiment [1] are: $\beta_{22}=1.03 / 0.97, \beta_{12}=1 / 0.97\left(\beta_{22}>\beta_{11}\right)$ and $\beta_{22}=0.97 / 1.03, \beta_{12}=1 / 1.03\left(\beta_{22}<\beta_{11}\right)$. The former case corresponds to the vortex in the spin state $\left\{F=1, m_{f}=-1\right\}$ of ${ }^{87} R b$ and the latter to the vortex in the state $\{2,2\}$. These two states will be called - state 1 and state 2 . It is clear that for $N=1$ Eq. (31) predicts instability for the vortex in the state 2 and stability for the vortex in the state 1, which supports results of the experimental observations [1].

Fig. 2 shows frequency resonance accompanied by the simultaneous mutual annihilation of excitations with positive and negative energies happening at some critical value of $\beta_{12}$, see Eq. (31). In fact it models transition from the situation with vortex in the state 1 to the case with vortex in the state 2 . Performing numerical studies for wide range of parameters, outside the validity region of analytical considerations, we have not been able to find regions of spectral instability of the unit vortex in state 1. Contrary, existence of instabilities of the vortex in state 2 due to exactly the same scenario, which is predicted in the weak interaction limit, can be readily demonstrated, see Fig. 3.

It is important to stress, that if two condensates are decoupled, $\beta_{12}=0$, then the negative energy mode $\vec{w}_{-1}^{-}$belongs to the vortex containing component and the positive energy mode $\vec{w}_{-1}^{+}$belongs to the vortex free component. Because of this separation instability is not possible for any values of $g$, which agrees with spectral stability of unit vortices in the singly component case reported in [6]. Thus we can conclude that the vortex instability in our example has essentially two-component nature and its analog may also exist in the case when second component is a non-condensate one.

Criterion similar to (31), but without corresponding energy analysis, has also been independently obtained in 11$]$ using two mode approach, i.e. condensate wave functions $\Psi_{1}$ and $\Psi_{2}$ have been presented as linear superposition of modes (24) with time dependent coefficients and GP equations have been reduced to the set of ordinary differential equations for these coefficients [11]. However, this method fails taking into account an eigenmode proportional to $r^{2}$, see $\vec{b}_{3}$ in (26), which makes an important contribution to the expressions for frequencies. Therefore Eqs. (27) and (31) are different from the corresponding results presented in [11].

To illustrate crossings and avoided crossings in the spectrum of unit vortices we show in Fig. 4 positive part of the frequency spectra of $\hat{\eta} \hat{\mathcal{H}}_{ \pm 1}$ and $\hat{\eta} \hat{\mathcal{H}}_{ \pm 2}$. One can see numerous points, which on the first glance can be interpreted as crossings in the frequency spectrum. However, under the close investigation those of them which are marked by the open circles, turns to be the avoided crossing of the excitations with equal energy signs, see Fig. 5. 

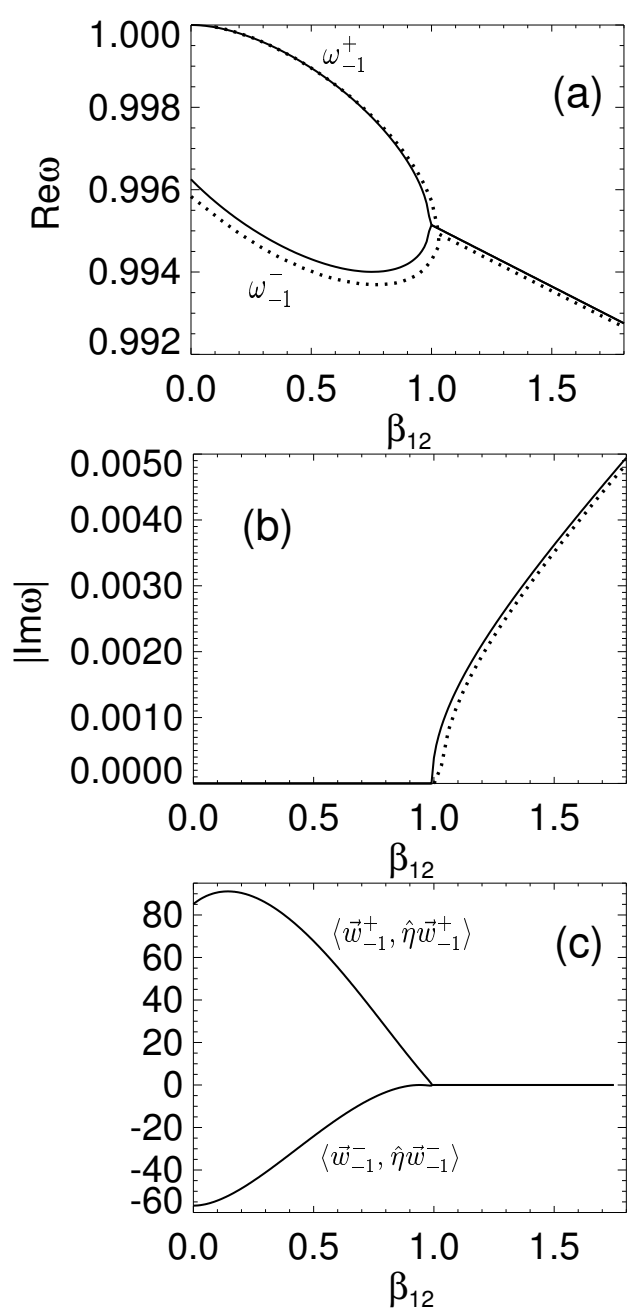

FIG. 2. (a),(b) Frequencies $\omega_{-1}^{ \pm}$vs $\beta_{12}: N=2, g=0.1, \beta_{22}=0.97 / 1.03$. (c) Inner products $\left\langle\vec{w}_{-1}^{ \pm}, \hat{\eta} \vec{w}_{-1}^{ \pm}\right\rangle$characterising energy signs of the corresponding excitations, see Eq. (30). Doted lines are numerical solutions of $\hat{\eta} \hat{\mathcal{H}}_{-1} \vec{w}_{l}=\omega_{l} \vec{w}_{l}$ and full lines correspond to Eqs. (28) and (30).

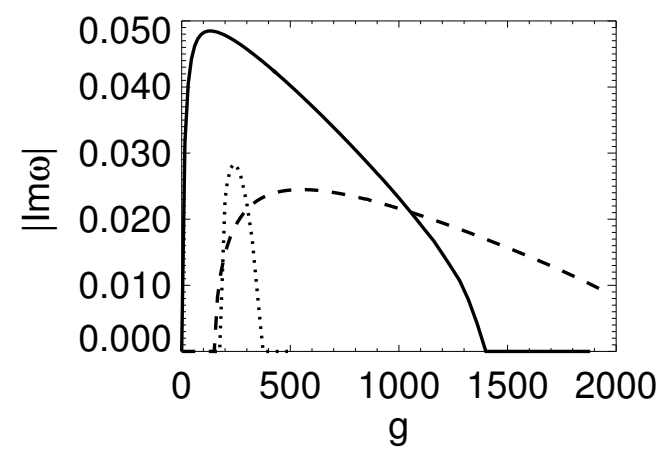

FIG. 3. Instability growth rate of the unit vortex in state 2 vs interaction parameter $g$. Full line $-N=1$; Dashed line $N=0.3 ;$ Dotted line $-N=9$. 


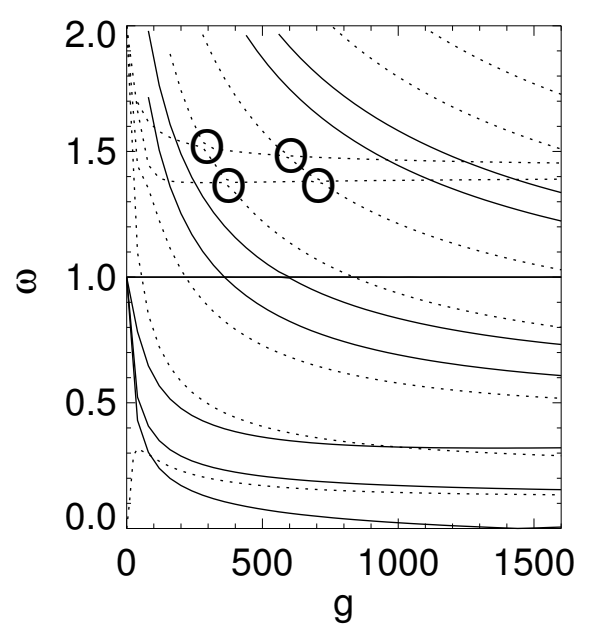

FIG. 4. Positive part of the frequency spectrum of the collective excitations with $|l|=1$ (full lines) and $|l|=2$ (doted lines) of the unit vortex in state 1 vs interaction parameter $g: N=1$. Open circles mark the avoided crossings (i.e. avoided frequency resonances) of the excitation with positive energies.

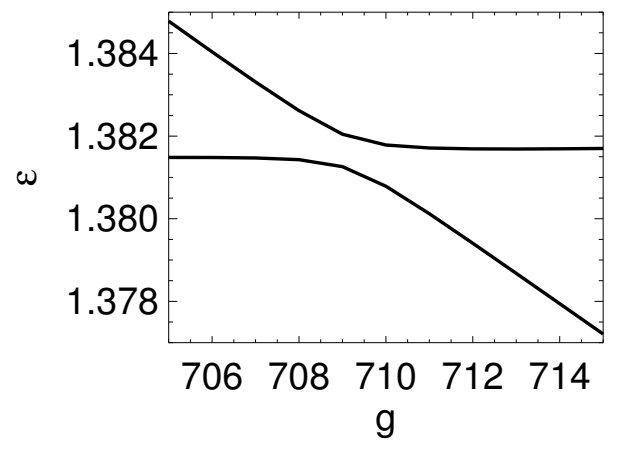

FIG. 5. Dependencies of energies of two elementary excitations with $l=2$ vs $g$ showing details of the rightmost from the avoided crossings marked in Fig. 4.

\section{DRIFT OF UNIT VORTICES}

The unstable modes of the vortex in the state 2 are of the dipole type, i.e. $|l|=1$, therefore their growth leads to the initial displacement of the vortex from the trap center. Displaced vortex carries less of the total angular momentum, compare to the momentum of the vortex positioned at the trap center. Lack of the angular momentum is compensated by two factors. First, vortex acquires a nonzero tangential velocity and therefore its trajectory is actually a spiral, which is similar to the dynamics of an optical vortex displaced from the center of a gaussian beam [44]. Second, angular momentum, and vortex itself, become gradually transferred into the second condensate component, which was first demonstrated in [10,11].

If drift instability is absent, then dissipative effects still can result in the vortex drift, which was predicted for the single component condensates by several authors [19.32. Presence of the second condensate opens a channel for the energy and angular momentum transfer from the vortex into the vortex free component. It can be seen that vector $\vec{b}_{1}$, being excited by the instability, provides a channel for this transfer. Thus drift instability can also be interpreted as due to dissipation of the energy and momentum by the vortex free condensate component.

In the limits $N \gg 1$ and $N \ll 1$ our model can be approximately considered as a single component condensate with $(N \gg 1)$ or without $(N \ll 1)$ vortex. Unit vortex and ground state of the single component condensate are known to be spectrally stable. Therefore drift instability disappears in both limits, see Fig. 3. Increase of $g$ for fixed $N$ also results in the suppression of the instability, see Fig. 3, which means that not only relative, but also absolute increase 
of the number of atoms in the vortex free component stabilizes the condensate.

\section{DRIFT AND SPLITTING OF HIGHER ORDER VORTICES}

Considering higher order vortices one can expect to find instability scenario resulting in their splitting into unit vortices. This scenarion is expected to be due to growth of the collective modes with $|l|>1$. However, as we will see below the drift instability linked to the dipole like modes, $|l|=1$, also can be presented. It leads to displacement of the whole vortex from the trap center without splitting, at least at the onset of the instability.

Both drift and splitting instabilities of the higher-order vortices appear as a result of the frequency resonances of the elementary excitations with negative and positive energies, similar to the case of unit vortices. $N \ll 1$ corresponds to the vortex free condensate and therefore both instabilities disappear in this limit. In the limit $N \gg 1$ only drift instability is suppressed and one can recover periodic in $N$ bands of the instabilities with complex frequencies and $|l|>1$ similar to the results reported for higher order vortices in a singly component condensate [9]. Note, that splitting itself was not explicitly demonstrated in []. It is also interesting to note that higher-order vortices in the free, $\hat{V}=0$, single component condensate are spectrally stable 45 . Thus splitting can be considered as induced by the trapping. The vortex free condensate component plays crucial role in the drift instability, but will the latter one be presented without trapping or not remains an open problem.

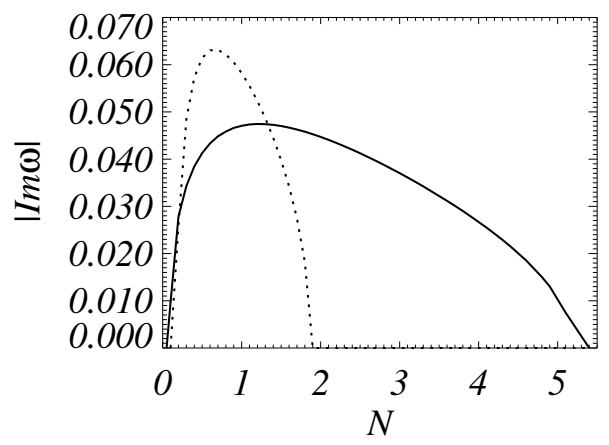

FIG. 6. Growth rate of the drift $|l|=1$ (full lines) and splitting $|l|=2$ (dotted lines) instabilities of the double vortex in the state 2 vs $N$ for $g=900$.

Double vortices: $L_{1}=0, L_{2}=2$

Considering double vortex we have found that it can be unstable with respect to the $|l|=1,2$ excitations. The vortex in the state 1 has been found surprisingly stable. One has to take relatively small values of $g$ and large $N$ to find splitting instability. Vortex in the state 2 is more unstable in a sense that splitting exists already for $N \sim 1$, see Fig. 6. As it is evident from Fig. 6 either drift or splitting instability can dominate vortex dynamics. If drift instability is dominant, then vortex first gets displaced from the trap center and only then splits into the unit ones, see Fig. 7. The latter happens due to the curved background which breaks cylindrical symmetry with respect to the vortex axis. After the splitting vortices remain close to each other and move towards the condensate periphery. Results of the numerical simulation of GP equations (1) presented in this Section were obtained starting from equilibrium states perturbed by random noise with amplitude $\sim 0.05 A_{1,2}$.

The dynamics is quite different when splitting instability is dominant, see Fig. 8. In this case unit vortices appear straight at the onset of the instability development and spiral out of the condensate center being always positioned symmetrically with respect to it. After a certain period of time vortices move back to the trap center and condensate state close to the initial one is restored, see Fig. 8, then the cycle is repeated with gradually worsening degree of periodicity.

During development of the instability angular momentum and vorticity become partially transferred into the second condensate. Analysis of the transverse profiles of the phases corresponding to the density profiles shown in Fig. 8, has revealed that black spots appearing in the second condensate are indeed unit vortices, not the density holes without topological structure. 

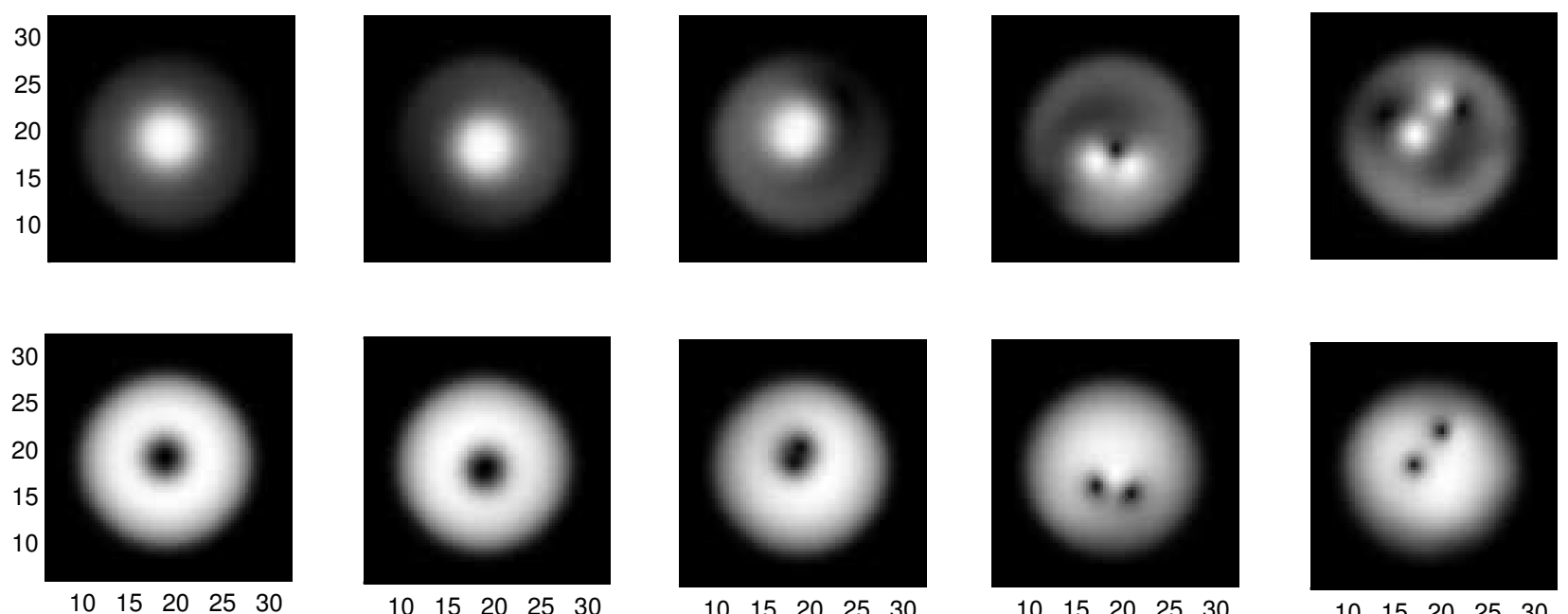

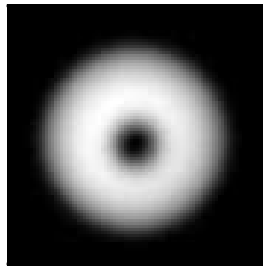

$10 \quad 1520 \quad 25 \quad 30$

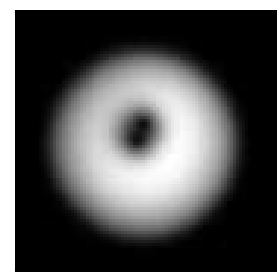

$\begin{array}{lllll}10 & 15 & 20 & 25 & 30\end{array}$

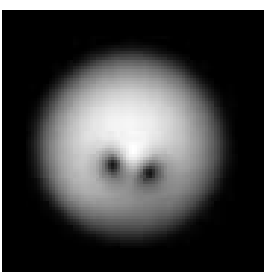

$\begin{array}{lllll}10 & 15 & 20 & 25 & 30\end{array}$

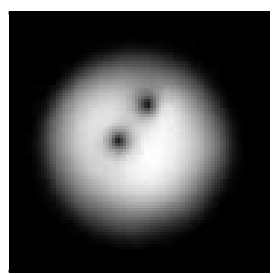

$\begin{array}{lllll}10 & 15 & 20 & 25 & 30\end{array}$

FIG. 7. Development of the drift instability of the double vortex in the state $2: g=900, N=2.5$. Top $-\left|\Psi_{1}(x, y)\right|^{2}$, bottom $-\left|\Psi_{2}(x, y)\right|^{2}$. Time interval between snapshots is 20. First snapshot corresponds to $t=60$.
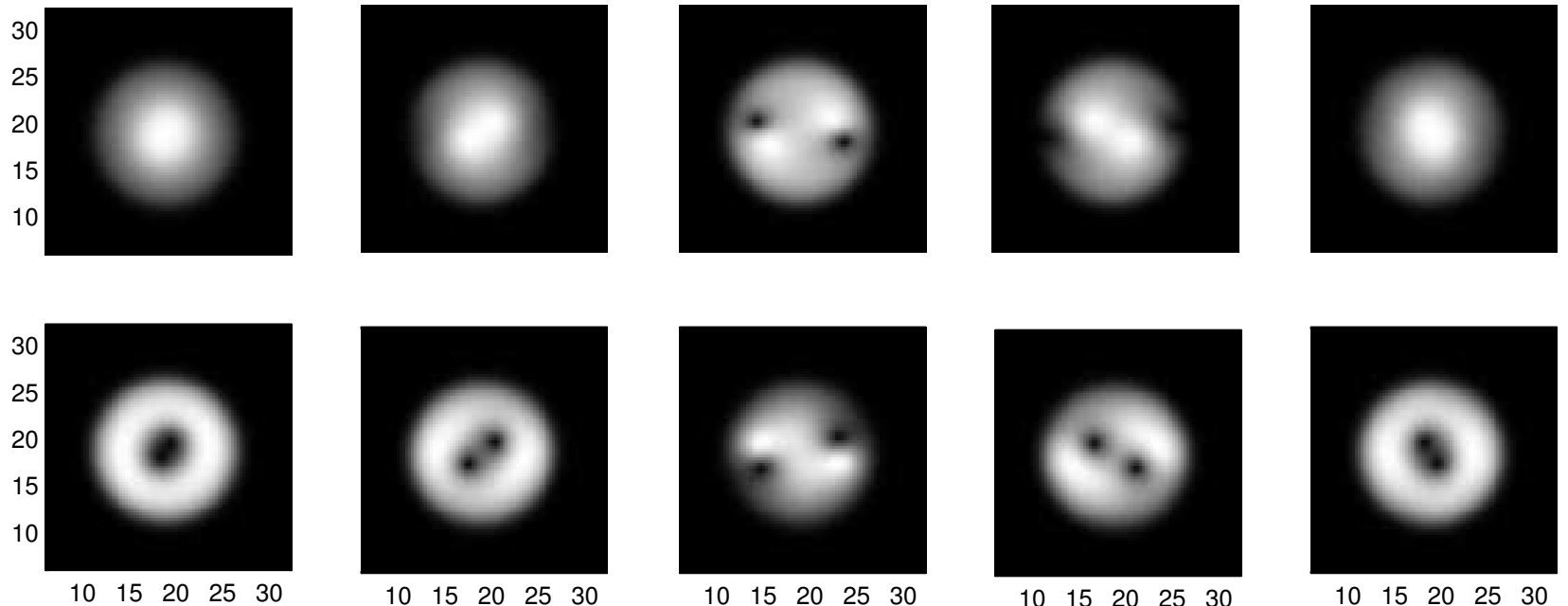

$\begin{array}{lllll}10 & 15 & 20 & 25 & 30\end{array}$

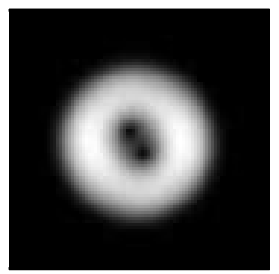

$\begin{array}{lllll}10 & 15 & 20 & 25 & 30\end{array}$

FIG. 8. Development of splitting instability of the double vortex in the state $2: g=900, N=0.7$. Top $-\left|\Psi_{1}(x, y)\right|^{2}$, bottom $-\left|\Psi_{2}(x, y)\right|^{2}$. Time interval between snapshots is 20. First snapshot corresponds to $t=30$.

Triple vortices: $L_{1}=0, L_{2}=3$

Reach variety of beautiful vortex lattices can be found considering instabilities of vortices of the order 3 and higher. This reachness can be understood in terms of spatial profiles of the unstable collective modes. E.g. triple vortex has been found unstable with respect to the perturbations with $|l|=1,2,3$. All components of the $|l|=2$ excitations are equal to zero at the trap center. Therefore one can expect that growth of this mode will develop into a spatial structure preserving vortex at the trap center. Contrary some of the components of the $|l|=3$ modes have humps at the center. Therefore their growth should repel all unit vortices out of the center, which leads to the breaking of the triple vortex into a triangular structure of the unit vortices moving away from the trap center. Both instabilities can be found for the same parameters values and can have close growth rates. Therefore the winning mode is selected 
through the process of complex competition, see Figs. 9,10.
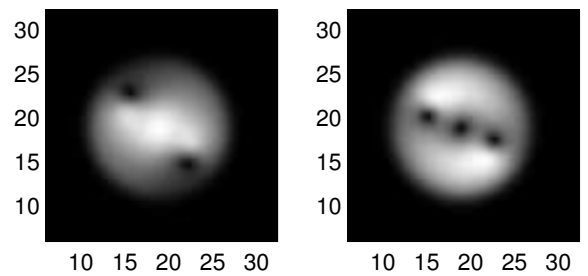

FIG. 9. $|l|=2$ instability of the triple vortex in the state $2: g=900, N=0.9, t=70$. Left $-\left|\Psi_{1}(x, y)\right|^{2}$, right $-\left|\Psi_{2}(x, y)\right|^{2}$.
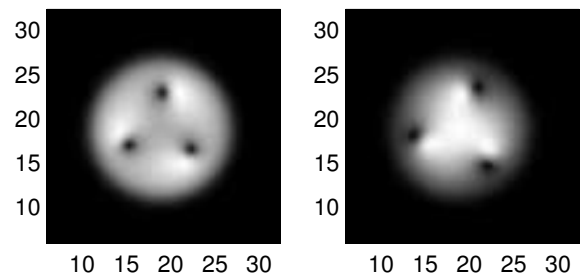

FIG. 10. $|l|=3$ instability of the triple vortex in the state $2: g=900, N=0.9, t=85$. Left $-\left|\Psi_{1}(x, y)\right|^{2}$, right $-\left|\Psi_{2}(x, y)\right|^{2}$.

\section{SUMMARY}

We have described general approach to stability of equilibrium in BEC using Bogoliubov theory and GP equation. Biorthogonality conditions (15), (16) and correspondence between frequency and energy spectra of the elementary excitations (20), (21) have been derived selfconsistently from first principals revealing several novel and conceptually important aspects originating in nonseldjoitness of the Bogoliubov operator.

It has been demonstrated that frequency resonances of the excitations with positive and negative energies can lead to their mutual annihilation and appearance of the collective modes with complex frequencies and zero energies. Conditions for the avoided crossing of energy levels have also been discussed. General theory has been verified both numerically and analytically in the weak interaction limit considering an example of vortices in a binary mixture of condensates.

Growth of excitations with complex frequencies leads to the two main scenarios of the instability development. First one is the spiraling of unit and double vortices out of the condensate center to its periphery. Second scenario is the splitting of the double and higher order vortices into unit ones. Absolute and/or relative increase of the number of particles in the vortex free condensate component have been found to have stabilizing effect.

\section{ACKNOWLEDGMENTS}

Author is particularly grateful to referees for their critical and very helpful comments. He also acknowledges discussions with S.M. Barnett and W.J. Firth. Numerical part of the work was significantly speeded up due to access to the computer equipment obtained via U.K. EPSRC grant GR/M31880 and assistance of G. Harkness and R. Martin.

[1] M.R. Matthews, B.P. Anderson, P.C. Haljan, D.S. Hall, C.E. Wieman, and E.A. Cornell, Phys. Rev. Lett. 83, 2498 (1999). 
[2] K.W. Madison, F. Chevy, W. Wohlleben, and J. Dalibard, Phys. Rev. Lett. 84, 806 (2000).

[3] J.E. Williams and M.J. Holland, Nature 401, 568 (1999).

[4] L.P. Pitaevskii, Zh. Exp. Teor. Fiz. 40, 646 (1961) [Sov. Phys. JETP 13, 451 (1961)].

[5] R.S. MacKay, Stability of equilibria of Hamiltonian systems, in 'Hamiltonian Dynamical Systems', eds. R.S. MacKay and J.D. Meiss (Hilger,Bristol,1987), page 137.

[6] N. Bogolubov, J. Phys. 11, 23 (1947).

[7] E.A. Goldstein and P. Meystre, Phys. Rev. A 55, 2935 (1997).

[8] E. Timmermans, Phys. Rev. Lett. 81, 5718 (1998).

[9] H. Pu, C.K. Law, J.H. Eberly, and N.P. Bigelow, Phys. Rev. A 59, 1533 (1999).

[10] J.J. Garcia-Ripoll and V.M. Pérez-Garsia, Phys. Rev. Lett. 84, 4264 (2000).

[11] J.J. Garcia-Ripoll and V.M. Pérez-Garsia, Phys. Rev. A 62, 033601 (2000).

[12] D.L. Feder, M.S. Pindoza, L.A. Collins, B.I. Schneider, and C.W. Clark, cond-mat/0004449.

[13] In the mathematical literature the energy sign is called signature of the eigenvalue or Krein sign.

[14] K. Weierstrass, in Weierstrass: Mathematishe Werke, vol. 1 (Berlin, 1894); reprinted (Hildesheim and New York: Olms and Johnson, 1967) page 233.

[15] A. Hasegawa, Plasma Instabilities and Nonlinear Effects (Springer, Berlin, 1975), page 18.

[16] R.S MacKay and P.G. Saffman, Proc. R. Soc. A 406, 115 (1986).

[17] M. Edwards, R.J. Dodd, C.W. Clark, P.A. Ruprecht, and K. Burnett, Phys. Rev. A 53, R1950 (1996).

[18] F. Dalfovo and S. Stringari, Phys. Rev. A 53, 2477 (1996).

[19] D.S. Rokhsar, Phys. Rev. Lett. 79, 2164 (1997).

[20] S. Sinha, Phys. Rev. A 55, 4325 (1997).

[21] R.J. Dodd, K. Burnett, M. Edwards, and C.W. Clark, Phys. Rev. A 56, 587 (1997).

[22] F. Zambelli and S. Stringari, Phys. Rev. Lett. 81, 1754 (1998).

[23] A.A. Svidzinsky and A.L. Fetter, Phys. Rev. A 58, 3168 (1998); A.A. Svidzinsky and A.L. Fetter, Phys. Rev. Lett. 84, 5919 (2000).

[24] E. Lundh, C.J. Pethick, and H. Smith, Phys. Rev. A 58, 4816 (1998).

[25] M. Linn and A.L. Fetter, Phys. Rev. A 60, 4910 (1999).

[26] D.L. Feder, C.W. Clark, and B. Schneider, Phys. Rev. Lett. 82, 4956 (1999).

[27] J.J. Garcia-Ripoll and V.M. Pérez-Garsia, Phys. Rev. A 60, 4864 (1999).

[28] D.L. Feder, C.W. Clark, and B. Schneider, Phys. Rev. A 61, 011601 (2000).

[29] B. Jackson, J.F. McCann, and C.S. Adams, Phys. Rev. A 61,013604 (2000).

[30] T. Isoshima and K. Machida, Phys. Rev. A 59, 2203 (1999).

[31] S. Stringari, Phys. Rev. Lett. 82, 4371 (1999).

[32] P.O. Fedichev and G.V. Shlyapnikov, Phys. Rev. A 60, R1779 (1999).

[33] A.L. Fetter, Ann. Phys. 70, 67 (1972).

[34] W.B. Colson, A.L. Fetter, J. Low Temp. Phys. 33, 231 (1978).

[35] T.-L. Ho and V.B. Shenoy, Phys. Rev. Lett. 77, 3276 (1996).

[36] C.K. Law, H. Pu, N.P. Bigelow, and J.H. Eberly, Phys. Rev. Lett. 79, 3105 (1997).

[37] B.D. Esry and C.H. Greene, Phys. Rev. A 59, 1457 (1999).

[38] P. Öhberg, Phys. Rev. A 59, 634 (1999).

[39] P. Noziéres and D. Pines, The Theory of Quantum Liquids. Vol. II. Superfluid Bose Liquid (Perseus Books, Cambridge, 1999), page 169.

[40] P.M. Morse and H. Feshbach, Methods of Theoretical Physics, Part 1 (McGraw-Hill, NY, 1953 ), page 884.

[41] W. Kohn, Phys. Rev. 123, 1242 (1961).

[42] J.F. Dobson, Phys. Rev. Lett. 73, 2244 (1994).

[43] D.V. Skryabin, Physica D 139, 186 (2000); D.V. Skryabin, Phys. Rev. E 60, 7511 (1999); D.V. Skryabin and W.J. Firth, Phys. Rev. E 58, R1252 (1998); ibid., 3916 (1998).

[44] Y.S. Kivshar, J. Christou, V. Tikhonenko, B. Luther-Davies, and L.M. Pismen, Opt. Commun. 152, 198 (1998).

[45] I. Aranson and V. Steinberg, Phys. Rev. B 53, 75 (1996). 